\title{
Synchronous and Subsequent Lesions of Serrated Adenomas and Tubular Adenomas of the Colorectum
}

\author{
T. Shimomura ${ }^{\text {a }}$ T. Hiyama ${ }^{d}$ S. Tanaka ${ }^{\text {b }}$ M. Yoshihara ${ }^{d}$ K. Arihiro ${ }^{c}$ \\ K. Chayama ${ }^{a}$ \\ Departments of a Gastroenterology and Metabolism, ${ }^{b}$ Endoscopy and ${ }^{\mathrm{C}}$ Anatomical Pathology, \\ Hiroshima University Hospital, and d Health Service Center, Hiroshima University, Higashihiroshima, Japan
}

\section{Key Words}

Serrated adenoma · Tubular adenoma · Synchronous lesion • Subsequent lesion

\begin{abstract}
The characteristics of synchronous and subsequent lesions of serrated adenomas (SAs) of the colorectum are still unclear. This study aimed to clarify the characteristics of synchronous and subsequent lesions of SAs compared with tubular adenomas (TAs) of the colorectum. Patients were divided into 2 groups: SA (127 patients) and TA (158 patients). The mean follow-up durations in the SA and TA groups were 39.7 and 42.7 months, respectively. The number and clinical features of the synchronous and subsequent lesions of both groups were examined. In the SA group, 19 (15\%) patients had synchronous lesions and $3(2 \%)$ patients had subsequent lesions. In the TA group, 68 (43\%) patients had synchronous lesions and 14 (9\%) patients had subsequent lesions. The frequencies of patients with synchronous and subsequent lesions in the $\mathrm{SA}$ group were significantly lower than those in the TA group $(p<0.0001$ and $p=0.02$, respectively). The most frequent synchronous lesion was SA (67\%) in the SA group and TA (95\%) in the TA group. The most subsequent lesion was SA (62\%) in the SA group and TA (100\%) in the TA group. The histology of the index polyp and synchronous and subsequent lesions tended to be identical.
\end{abstract}

No invasive colorectal carcinomas were observed in either group. Our data suggest that the colonic tumorigenesis potential of patients with SA may differ from that of patients with TA.

Copyright $\odot 2010$ S. Karger AG, Basel

\section{Introduction}

Serrated adenomas (SAs) of the colorectum are recognized as a distinct form of neoplasia, histologically defined by the presence of hyperplastic and adenomatous features as proposed by Longacre and Fenoglio-Preiser [1] in 1990. Histological diagnosis of SA is made by observation of a serrated glandular pattern, the presence of goblet cell immaturity and upper zone mitoses, the prominence of nucleoli, and the absence of a thickened collagen table. SAs harbor malignant potential, and high-grade dysplasia has been observed in $11 \%$ of SAs $[1,2]$.

Recently, the clinical and molecular differences between SAs and conventional tubular adenomas (TAs) have been detected. Clinically, SAs are located predominantly in the sigmoid colon and rectum, and incidences of high-grade dysplasia differ between SAs and TAs [3]. Genetically, SAs have frequent mutations of $p 53$, K-ras, and B-raf as well as methylations of $h M L H 1, p 16$, and IGFBP7 [4]. However, research on synchronous and sub- 

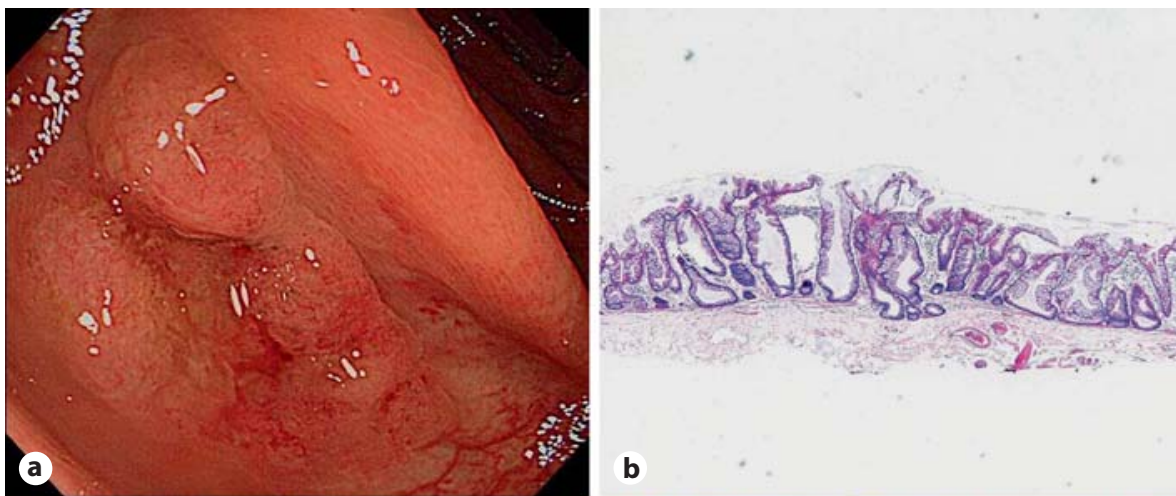

Fig. 1. Colonoscopic and microscopic findings of serrated adenoma. a Conventional colonoscopic finding. A sessile polyp is seen in the ascending colon. $\mathbf{b}$ Microscopic finding $($ HE. $\times 40)$. The serrated pattern of the crypt is shown. c Microscopic finding (HE. × 200). Pseudoinvasion of the tumor into the submucosa is observed. d Microscopic finding $(\mathrm{HE} . \times 400)$. The nuclei show atypia.
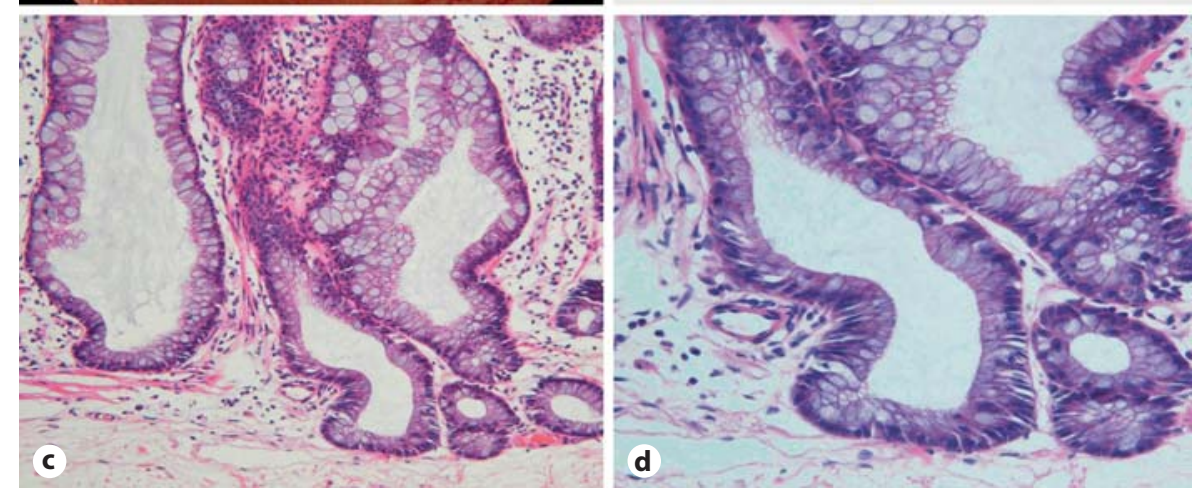

sequent lesions of SA is quite limited. To our knowledge, there is only 1 study on the issue at present [5]. Thus, the characteristics of those lesions are still unclear. The purpose of this study was to clarify the characteristics of synchronous and subsequent lesions of SAs compared to those of TAs.

\section{Patients and Methods}

This is a retrospective study, and the patients described in this paper were diagnosed from their first colonoscopy by polypectomy or endoscopic mucosal resection via endoscope report files and pathology database files collected between January 2003 and December 2008 at Hiroshima University Hospital (Hiroshima, Japan). The cases that fulfilled the following criteria were selected for this study and were classified into the SA and TA groups: (1) underwent complete colonoscopy, (2) polyp(s) were resected by polypectomy or endoscopic mucosal resection for full evaluation, (3) diagnosed as SA and/or TA at the initial colonoscopic examination, (4) followed up after at least 1 year and had at least 1 followup colonoscopy, (5) if only 1 SA or TA was present, the polyp was regarded as an index polyp, and (6) if synchronous SA and/or TA was present, the largest polyp was regarded as the index polyp; patients with polyposis syndromes such as familial adenomatous polyposis and hyperplastic/serrated polyposis (HSP), those who had undergone colectomy, and those with advanced colorectal cancer (CRC) were excluded because of the subsequent surgical treatment. All of the SA and TA diagnoses were confirmed by a pathologist (K.A.). The location of the lesion was divided into proximal (from the cecum to the splenic flexure) and distal (from the descending colon to the rectum) regions.

We identified 127 patients in the SA group, and 158 randomly selected age- and sex-matched patients were enrolled into the TA group. The mean follow-up duration was 39.7 months (range 1358 ) in the SA group and 42.7 months (range 14-62) in the TA group. We investigated the number and clinicopathologic features of synchronous and subsequent lesions in both groups.

Statistical analysis was evaluated using a $\chi^{2}$ test. $p<0.05$ was considered statistically significant.

\section{Results}

In the SA group, the male/female ratio was $86 / 41$ and the mean age at the first diagnosis was 63.4 years (range $36-91)$. In the TA group, the male/female ratio was 116/42 and the mean age at the first diagnosis was 65.3 years (range 38-88) (table 1; fig. 1).

In the SA group, 19 (15\%) patients had synchronous lesions and $3(2 \%)$ patients had subsequent lesions in the follow-up period (table 1). In the TA group, 68 (43\%) patients had synchronous lesions and 14 (9\%) patients had subsequent lesions in the follow-up period. The frequencies of patients with synchronous and subsequent lesions 
Table 1. Frequencies of patients with synchronous and subsequent lesions in the SA and TA groups

\begin{tabular}{|c|c|c|c|}
\hline & $\begin{array}{l}\text { SA group } \\
(\mathrm{n}=127)\end{array}$ & $\begin{array}{l}\text { TA group } \\
(\mathrm{n}=158)\end{array}$ & $\begin{array}{l}\mathrm{p} \\
\text { value }\end{array}$ \\
\hline Male/female ratio & $86 / 41$ & $116 / 42$ & NS \\
\hline Mean age (range), years & $63.4(36-91)$ & $65.3(38-88)$ & NS \\
\hline $\begin{array}{l}\text { Patients with synchro- } \\
\text { nous lesions, } \mathrm{n}\end{array}$ & $19(15 \%)$ & $68(43 \%)$ & $<0.0001$ \\
\hline Patients with & & & \\
\hline subsequent lesions, $\mathrm{n}$ & $3(2 \%)$ & $14(9 \%)$ & 0.02 \\
\hline
\end{tabular}

Table 2. Histology of synchronous lesions in the SA and TA groups

\begin{tabular}{lcrlr}
\hline & SA & TA & HP & \multicolumn{1}{l}{ Total } \\
\hline SA group $(\mathrm{n}=19)$ & $58(67)$ & $12(14)$ & $17(19)$ & $87(100)$ \\
TA group $(\mathrm{n}=68)$ & 0 & $187(95)$ & $9(5)$ & $196(100)$
\end{tabular}

Figures in parentheses are percentages.

in the SA group were significantly lower than those in the TA group $\left(\mathrm{p}<0.0001\right.$ and $\mathrm{p}=0.02$ by $\chi^{2}$ test, respectively).

As for synchronous lesions, a total of 87 and 196 lesions were detected in the SA and TA groups, respectively (table 2). Interestingly, the most frequent synchronous lesion was SA (67\%) in the SA group, and TA (95\%) in the TA group. Thus, the histology of the index polyp and synchronous lesions was identical in most patients. No invasive colorectal carcinomas were observed in either group.

As for subsequent lesions, a total of 8 and 22 lesions were detected in the SA and TA groups, respectively (table 3). No invasive CRCs were observed in either group. The most frequent subsequent lesion was SA (62\%) in the SA group and TA (100\%) in the TA group. Thus, similar to synchronous lesions, the histology of the index polyp and subsequent lesions was identical in most patients.

The locations of the synchronous and subsequent lesions of both groups are indicated in table 4 . The distribution pattern did not differ significantly between the SA and TA groups.

Synchronous and Subsequent Lesions of SA
Table 3. Histology of subsequent lesions in the SA and TA groups

\begin{tabular}{llclr}
\hline & SA & TA & HP & \multicolumn{1}{c}{ Total } \\
\hline SA group $(\mathrm{n}=3)$ & $5(62)$ & $3(38)$ & 0 & $8(100)$ \\
TA group $(\mathrm{n}=14)$ & 0 & $22(100)$ & 0 & $22(100)$ \\
\hline
\end{tabular}

Figures in parentheses are percentages.

Table 4. Locations of synchronous and subsequent lesions of the SA and TA groups

\begin{tabular}{lrrl}
\hline & Proximal & Distal & p value \\
\hline SA group & & & NS \\
SA & 32 & 31 & \\
TA & 11 & 4 & \\
HP & 5 & 12 & NS \\
TA group & & & \\
SA & 0 & 0 & \\
TA & 106 & 103 & \\
HP & 5 & 4 & \\
\hline
\end{tabular}

NS $=$ Not significant

\section{Discussion}

In the present study, we showed that the colonic tumorigenesis potential of patients with SA may differ from that of patients with TA. The frequencies of synchronous and subsequent lesions in the SA group were significantly lower than those in the TA group. In addition to this, the most frequent synchronous and subsequent lesions were SAs in the SA group and TAs in the TA group.

The frequency of SAs has been reported to be $1-2.4 \%$ of all colorectal polyps, indicating that SAs are rare polyps in the colorectum $[1,6]$. However, $22.6 \%$ of rectosigmoid polyps originally diagnosed as hyperplastic polyps (HPs) demonstrated features of SAs upon reanalysis [7]. In a recent study, the proportion of SAs among all index polyps was $10 \%$ [8]. It has also been reported that serrated adenocarcinoma arising from SA accounts for at least $5.8 \%$ of CRCs [5]. These data suggest that SAs may not be rare lesions but rather lesions of significant importance.

The grade of malignant potential of SAs is of great interest. To evaluate the malignant potential, examination 
of the incidence of malignant lesions including highgrade dysplasia and carcinoma in SAs is one method. Song et al. [7] compared the clinicopathologic features of 124 SAs from 116 patients and 419 TAs from 200 randomly selected patients. The incidence of malignant lesions including high-grade dysplasia and carcinoma in SA was found to be lower than in TA $(3.2$ vs. $9.3 \%$, p < 0.05). Iwabuchi et al. [3] reported that the percentage of concomitant carcinomas in cases of SA was significantly lower than in TA. These data suggest that SAs may be premalignant lesions but have a lower malignant potential than TAs.

Examining synchronous and subsequent lesions of SA compared with those of TA is another method to evaluate the malignant potential of SA. However, there are few reports on the characteristics of synchronous and subsequent lesions of SAs compared with those of TAs. To our knowledge, there is only 1 study on the issue at present. Lazarus et al. [5] reported that synchronous lesions identified at the index colonoscopy were of the same histological type as the index polyp ( $87 \%$ of the SA group and $94 \%$ of the TA group), and subsequent lesions were also of the same histological type as the index polyp ( $75 \%$ of the SA group and 70\% of the TA group), which is similar to our results. These findings suggest that the genetic and environmental factors determining the predominant type of polyp may influence the histology of colorectal polyps. In addition, the same authors reported that the frequencies of synchronous and subsequent lesions in the SA group were significantly lower than those in the TA group, which is also similar to our results.

Serrated lesions of the colorectum are subclassified primarily into 3 subtypes: HP, traditional serrated adenoma (TSA), and sessile serrated adenoma/polyp (SSA/P). SSA/P have architectural features that include dilatation of the base of the crypts, often with crypts that seem to grow parallel to the muscularis mucosa and result in T- or L-shaped crypts. TSA also has serration but with a uniform population of dysplastic epithelial cells that are columnar cells with eosinophilic cytoplasm, have a villiform configuration, and appear protuberant rather than sessile [4]. In the present study, SA included both TSA and SSA/P. Although differences in clinicopathologic and molecular characteristics between these lesions have been reported, histologic diagnosis of these lesions is still confused among pathologists at present. There are some differences in diagnosis of TSA, SSA/P, and HP among pathologists. In addition to this, the number of lesions examined in the present study was relatively limited. Therefore, we did not subclassify SAs in the present study.
Of course, further examinations with subclassification of SAs may be needed to clarify the differences between TSA and SSA/P.

It has been suggested that left- and right-sided colorectal cancers differ in their associated genetic alterations in neoplastic transformation, based on studies in Western countries. For example, microsatellite instability-positive (MSI+) cancers are preferentially found in right-sided colon cancers and in older women. Previous studies have indicated a higher incidence of $\mathrm{CpG}$ island methylator phenotype-positive (CIMP+) tumors in right-sided colon cancers compared with left-sided colon cancers. On the other hand, chromosomal instability is said to characterize left-sided colorectal cancers. These findings have provided further evidence of the existence of at least 2 mechanisms of colorectal cancer pathogenesis. One group (CIMP+/MSI + ) occurs predominantly on the right side of the colon, and the other (chromosomal instability) occurs predominantly on the left side of the colon.

There are several studies on genetic alterations of SAs, e.g. the presence of B-raf or K-ras mutations [9], extensive DNA methylations [10], and the inactivation of hMLH1 or MGMT [10, 11]. We previously reported frequent $p 53$ mutations in SAs [12]. Recently, serrated pathways have been proposed as one of the carcinogenetic pathways of the colorectum and are divided into 2 categories: $\mathrm{CIMP}+/$ MSI+ cancer with a B-raf mutation and CIMP+/MSIwith a B-raf mutation [4]. SSA/P is most often a rightsided lesion characterized by a B-raf mutation, whereas TSA is most often left-sided with a K-ras mutation [13]. The B-raf mutation is commonly seen in CIMP+/MSIcancers (44\%), though at a lower frequency than in CIMP+/MSI+ cancers (94\%), and MSI+ cancers occur predominantly in the proximal colon. SSA/P is likely to be the principal precursor of MSI+ colorectal cancer [14].

We excluded patients with polyposis syndromes, such as familial adenomatous polyposis and HSP, from this study. HSP, however, is a distinctive entity. The definition of HSP is as follows: the presence of (1) $>30 \mathrm{HPs} / \mathrm{SAs}$ distributed throughout the colon, (2) $>5 \mathrm{HPs} / \mathrm{SAs}$, proximal to the sigmoid colon, of which at least 2 polyps are $>1 \mathrm{~cm}$ in size, or (3) any number of HPs/SAs located proximal to the sigmoid colon in a patient with a first-degree relative with HSP [15]. In the case of HSP, Hyman et al. [16] reported that 7 of 13 (54\%) patients with HSP developed CRC during the follow-up period. Rubio et al. [17] summarized 30 publications about HSP from 1977 to 2005 and stated that $51 \%(67 / 131)$ of the HSP patients had CRC at the time of diagnosis of HSP and/or during the followup period. This percentage is very high when compared 
with that of a recent study of 1,552 consecutive colorectal adenomas where only $1.7 \%(27 / 1,552)$ showed invasive carcinoma [18] and which reported that the overall lifetime cumulative incidence of CRC in a population is only $5.5 \%[18,19]$. Several researchers have postulated that, in HSP patients, carcinomas may evolve through the HP-SA sequence $[20,21]$. Although patients with SA seem not to be at a high risk for CRC development, patients with HSP seem to be at a very high risk for CRC development. The prevalence of HSP is still unclear, and this point should also be investigated.

In conclusion, our data suggest that the colonic tumorigenesis potential of patients with SA may differ from that of patients with TA. A large-scale prospective study on the synchronous and subsequent lesions of SAs and TAs of the colorectum should be performed to confirm the results.

\section{References}

$\checkmark 1$ Longacre TA, Fenoglio-Preiser CM: Mixed hyperplastic adenomatous polyps/serrated adenomas: a distinct form of colorectal neoplasia. Am J Surg Pathol 1990;14:524-537.

$\checkmark 2$ Harvey NT, Ruszkiewicz A: Serrated neoplasia of the colorectum. World J Gastroenterol 2007;13:3792-3798.

-3 Iwabuchi M, Sasano H, Hiwatashi N, et al: Serrated adenoma: a clinicopathological, DNA ploidy, and immunohistochomical study. Anticancer Res 2000;20:1141-1147.

4 Leggett B, Whitehall V: Role of the serrated pathway in colorectal cancer pathogenesis. Gastroenterology 2010;138:2088-2100.

5 Lazarus R, Juunttila OE, Karttunen TJ, et al: The risk of metachronous neoplasia in patients with serrated adenoma. Am J Clin Pathol 2005;123:349-359.

-6 Bariol C, Hawkins NJ, Turner JJ, et al: Histopathological and clinical evaluation of serrated adenomas of the colon and rectum. Mod Pathol 2003;16:417-423.

-7 Song SY, Kim YH, Yu MK, et al: Comparison of malignant potential between serrated adenomas and traditional adenomas. J Gastroenterol Hepatol 2007;22:1786-1790.

8 Goldstein NS, Bhanot P, Odish E, et al: Hyperplastic-like colon polyps that preceded microsatellite-unstable adenocarcinomas. Am J Clin Pathol 2003;119:778-796.
Chan TL, Zhao W, Leung SY, et al: BRAF and KRAS mutations in colorectal hyperplastic polyps and serrated adenomas. Cancer Res 2003;63:4878-4881.

10 Park SJ, Rashid A, Lee JH, et al: Frequent CpG island methylation in serrated adenomas of the colorectum. Am J Pathol 2003; 162:815-822.

11 Jass JR, Young J, Leggett BA: Hyperplastic polyps and DNA microsatellite unstable cancers of the colorectum. Histopathology 2000;37:295-301.

12 Hiyama T, Yokozaki H, Shimamoto F, et al: Frequent $p 53$ gene mutations in serrated adenomas of the colorectum. J Pathol 1998;186: 131-139.

13 Torlakovic EE, Gomez JD, et al: Sessile serrated adenoma (SSA) vs. traditional serrated adenoma (TSA). Am J Surg Pathol 2008;32: 21-29.

14 Kambara T, Simms LA, et al: BRAF mutation is associated with DNA methylation in serrated polyps and cancers of the colorectum. Gut 2004;53:1137-1144.

15 Burt RW, Jass J: Hyperplastic polyposis; in Hamilton SR, Aaltonen LA (eds): World Health Organisation Classification of Tumours Pathology and Genetics. Berlin, Springer, 2000, pp 135-136.
6 Hyman NH, Anderson P, Blasyk H: Hyperplastic polyposis and the risk of colorectal cancer. Dis Colon Rectum 2004;47:21012104.

17 Rubio CA, Stemme S, Jaramillo E, et al: Hyperplastic polyposis coli syndrome and colorectal carcinoma. Endoscopy 2006;38: 266-270.

18 Rubio CA, Nesi G, Messerini L, et al: Serrated and microtubular colorectal adenomas in Italian patients: a 5-year survey. Anticancer Res 2005;25:1353-1359.

19 Atkin W, Saunders B. Surveillance guidelines after removal of colorectal adenomatous polyps. Gut 2002;51(suppl 5):v6-v9.

20 Shimamoto F, Tanaka S, Tahara E: Pathogenesis of serrated adenoma of the colorectum: implication for malignant progression; in Tahara E (ed): Molecular Pathology of Gastroenterological Cancer. Tokyo, Springer, 1997, pp 93-106

21 Oka S, Tanaka S, Hiyama T, et al: Clinicopathologic and endoscopic features of colorectal serrated adenoma: differences between polypoid and superficial types. Gastrointest Endosc 2004;59:213-219. 